\title{
Analytic Neutrino Oscillation Probabilities in Matter: Revisited
}

\author{
Stephen J. Parke ${ }^{* \dagger}$ \\ Theoretical Physics Department, Fermi National Accelerator Laboratory, \\ Batavia, IL 60510, USA \\ E-mail: parkeefnal.gov \\ Peter B. Denton \\ Niels Bohr International Academy, Niels Bohr Institute, University of Copenhagen, \\ Blegdamsvej 17, 2100, Copenhagen, Denmark \\ E-mail: peterbd1@gmail.com

\section{Hisakazu Minakata} \\ Instituto Física Teórica, UAM/CSIC, Cantoblanco E-28049 Madrid, Spain \\ \& Research Center for Cosmic Neutrinos, ICRR, University of Tokyo, Japan \\ E-mail: hisakazu.minakata@gmail.com
}

\begin{abstract}
We summarize our recent paper on neutrino oscillation probabilities in matter, explaining the importance, relevance and need for simple, highly accurate approximations to the neutrino oscillation probabilities in matter. Simple expressions for the neutrino mixing angles and mass squared differences in matter are given in an Appendix. Using these in the vacuum oscillation probabilities instead of the vacuum values, gives an excellent approximation to the oscillation probabilities in matter.
\end{abstract}

The 19th International Workshop on Neutrinos from Accelerators-NUFACT2017

25-30 September, 2017

Uppsala University, Uppsala, Sweden

\footnotetext{
* Speaker.

$\dagger$ I wish to thank the organizers for the fabulous Swedish hospitality!
} 


\section{Neutrino Propagation in Matter}

The evolution of a neutrino flavor state in matter is given by

$$
i \frac{d}{d x} v=H v \text { with } v=\left(\begin{array}{c}
v_{e} \\
v_{\mu} \\
v_{\tau}
\end{array}\right) \text { and } H=\frac{1}{2 E}\left\{U\left[\begin{array}{ccc}
0 & 0 & 0 \\
0 & \Delta m_{21}^{2} & 0 \\
0 & 0 & \Delta m_{31}^{2}
\end{array}\right] U^{\dagger}+\left[\begin{array}{ccc}
a(x) & 0 & 0 \\
0 & 0 & 0 \\
0 & 0 & 0
\end{array}\right]\right\} \text {, }
$$

where "a" is the matter potential, $a=2 \sqrt{2} G_{F} N_{e} E$.

We have developed a single perturbative expansion for the oscillation probabilities in constant matter that satisfies the following criteria, see [1];

1. valid and accurate for all baseline divided by neutrino energy and all values of matter potential, i.e. over the full $(L / E, Y \rho E)$ plane,

2. has the universal form for the L/E dependence of the oscillation probabilities i.e. three sin squared terms and a $\mathrm{CP}$ violating triple sin term,

3. since the atmospheric and solar resonance have to be dealt with in a non-perturbative fashion, we need to use $\sqrt{\cdots}$ function but will use nothing more complex ${ }^{1}$,

4. the form of the mass eigenvalues squared in matter is particularly simple which leads to simple forms for the mixing angles in matter at zeroth order, providing an enhanced understand of oscillation probabilities in matter.

Our perturbative expansion should be compared to other perturbative expansions in the literature, see [3], which do not satisfy all of the above criteria.

To develop a perturbation theory for the neutrino mass squared's in matter as well as the elements of MNS in matter, we first need to take care of the resonance regions non-perturbatively. We start by splitting the Hamiltonian, $H$, into two pieces, the diagonal part, $H_{0}$, and the nondiagonal part $H_{1}$ such that $H=H_{0}+H_{1}$ :

$$
H_{0}=\frac{1}{2 E}\left[\begin{array}{lll}
\lambda_{a} & & \\
& \lambda_{b} & \\
& & \lambda_{c}
\end{array}\right] \quad \text { where }\left\{\begin{aligned}
\lambda_{a} & \equiv a+s_{13}^{2} \Delta m_{e e}^{2} \\
\lambda_{b} & \equiv\left(c_{12}^{2}-s_{12}^{2}\right) \Delta m_{21}^{2} \\
\lambda_{c} & \equiv c_{13}^{2} \Delta m_{e e}^{2}
\end{aligned}\right.
$$

where $\Delta m_{e e}^{2} \equiv \Delta m_{31}^{2}-s_{12}^{2} \Delta m_{21}^{2}=\Delta m_{32}^{2}+c_{12}^{2} \Delta m_{21}^{2}$. Note, $\lambda_{a}, \lambda_{b}$ and $\lambda_{c}$ are the asymptotic values ${ }^{2}$ of the mass squared eigenstates as $|a| \rightarrow \infty$, including terms of $\mathrm{O}\left(a^{0}\right)$.

The non-diagonal part, $H_{1}$, is given by

$$
H_{1}=s_{13} c_{13} \frac{\Delta m_{e e}^{2}}{2 E}\left[\begin{array}{c}
{ }_{1} \\
0 \\
1
\end{array}\right]+c_{13} s_{12} c_{12} \frac{\Delta m_{21}^{2}}{2 E}\left[\begin{array}{cc}
1 & \\
1 & 0 \\
0 & 0
\end{array}\right]-s_{13} s_{12} c_{12} \frac{\Delta m_{21}^{2}}{2 E}\left[\begin{array}{cc}
0 \\
0 & 1 \\
1
\end{array}\right] \text {. }
$$

Given that

$$
s_{13} c_{13} \sim 0.15, \quad c_{13} s_{12} c_{12}\left(\Delta m_{21}^{2} / \Delta m_{e e}^{2}\right) \sim 0.015 \quad \text { and } \quad s_{13} s_{12} c_{12}\left(\Delta m_{21}^{2} / \Delta m_{e e}^{2}\right) \sim 0.002,
$$

\footnotetext{
${ }^{1}$ Compared to the exact results which involve the $\cos \left[\frac{1}{3} \arccos (\cdots)\right]$ expressions that appear in [2].
}

${ }^{2}$ The constant $s_{12}^{2} \Delta m_{21}^{2}$ has been subtracted from all eigenvalue compared to [1]. 
there is a hierarchy in the size of the three terms in $H_{1}$. So it is natural to perform first a rotation in the 1-3 space followed by a rotation in the 1-2 space. After, these rotations, the level crossings that existed in $H_{0}$ of eqn 1.1, as one varied the matter potential, no longer exist in the new $H_{0}$ given by:

$$
H_{0}=\frac{1}{2 E}\left[\begin{array}{lll}
\lambda_{1} & & \\
& \lambda_{2} & \\
& & \lambda_{3}
\end{array}\right] \quad \text { and } \quad H_{1}=\sin \left(\phi-\theta_{13}\right) s_{12} c_{12} \frac{\Delta m_{21}^{2}}{2 E}\left[\begin{array}{ccc}
0 & 0 & -s_{\psi} \\
0 & 0 & c_{\psi} \\
-s_{\psi} & c_{\psi} & 0
\end{array}\right]
$$

where $\lambda_{i}$ are the square of the neutrino mass in matter and $\phi, \psi$ are the mixing angles $\theta_{13}, \theta_{12}$ in matter. Note, in vacuum, $\sin \left(\phi-\theta_{13}\right)=0$ so that $H_{1}=0$. Expressions for $\lambda_{i}, \phi$ and $\psi$ will be given in next section.

At this point, one could perform a further rotation. For NO, if one performs an additional rotation in the 1-3 space, then the new $H_{1}$ will be proportional to $\sin \left(\phi-\theta_{13}\right) c_{\psi}$ whose magnitude for all "a" is $<s_{13}$. Similarly for IO, if the rotation is performed in the 2-3 space and the new $H_{1}$ wiil be proportional to $\sin \left(\phi-\theta_{13}\right) s_{\psi}$. These rotations would significantly improve the 0 th order approximation especially in the region where $\sin \left(\phi-\theta_{13}\right) \sim c_{13}$.

Instead, to keep one perturbative expansion for both mass orderings, we will do perturbation theory using the results of the first two rotations, i.e. eqn 1.3.

\section{A Simple, Accurate Method for Calculate Oscillation Probabilities in Matter}

A simple and accurate way to evaluate oscillation probabilities, see [1], is given in this section. Details as to the why's and how's of this method are contained in this paper.

After performing a rotation in the 1-3 space:

$$
\begin{aligned}
\lambda_{0}=\lambda_{b}, \quad \lambda_{ \pm} & =\frac{1}{2}\left(\lambda_{c}+\lambda_{a} \pm \operatorname{sign}\left(\Delta m_{e e}^{2}\right) \sqrt{\left(\lambda_{c}-\lambda_{a}\right)^{2}+\left(2 s_{13} c_{13} \Delta m_{e e}^{2}\right)^{2}}\right) \\
& =\frac{1}{2}\left(\Delta m_{e e}^{2}+a \pm \operatorname{sign}\left(\Delta m_{e e}^{2}\right) \sqrt{\left(\Delta m_{e e}^{2} \cos 2 \theta_{13}-a\right)^{2}+\left(2 s_{13} c_{13} \Delta m_{e e}^{2}\right)^{2}}\right) \\
\sin \phi & =\sqrt{\frac{\lambda_{+}-\lambda_{c}}{\lambda_{+}-\lambda_{-}}} \quad \text { with } 0 \leq \phi \leq \pi / 2,
\end{aligned}
$$

which satisfies

$$
\phi(a)=\frac{\pi}{2}-\phi\left(2 \Delta m_{e e}^{2} \cos \theta_{13}-a\right) \quad \text { and } \quad \phi_{N O}(a)=\phi_{I O}(-a) .
$$

$\phi$ is $\theta_{13}$ in matter and $\left(\lambda_{a}, \lambda_{b}, \lambda_{c}\right) \rightarrow\left(\lambda_{-}, \lambda_{0}, \lambda_{+}\right)$. When $\left|\lambda_{c}-\lambda_{a}\right| \gg 2 s_{13} c_{13}\left|\Delta m_{e e}^{2}\right|$ then $\left(\lambda_{-}, \lambda_{+}\right) \approx$ $\left(\lambda_{a}, \lambda_{c}\right)$ or $\left(\lambda_{c}, \lambda_{a}\right)$. If $a=0, \phi=\theta_{13}$ and $\left(\lambda_{-}, \lambda_{0}, \lambda_{+}\right)=\left(0,\left(c_{12}^{2}-s_{12}^{2}\right) \Delta m_{21}^{2}, \Delta m_{e e}^{2}\right)$.

It is simple to show that

$$
\sin \left(\phi-\theta_{13}\right)=\operatorname{sign}\left(a \Delta m_{e e}^{2}\right) \sqrt{\left(\lambda_{-}-a c_{\phi}^{2}\right) / \Delta m_{e e}^{2}} \approx s_{13} c_{13}\left(a / \Delta m_{e e}^{2}\right)+\mathscr{O}\left[\left(a / \Delta m_{e e}^{2}\right)^{2}\right],
$$

which ultimately will determine the size of the perturbing Hamiltonian. Note, that for a neutrino energy of $3 \mathrm{GeV}$ and earth crust density $\sin \left(\phi-\theta_{13}\right) \approx 0.04$. 
Then, performing a rotation in the 1-2 space: with $\lambda_{3}=\lambda_{+}$and

$$
\begin{aligned}
\lambda_{2,1} & =\frac{1}{2}\left(\lambda_{0}+\lambda_{-} \pm \sqrt{\left(\lambda_{0}-\lambda_{-}\right)^{2}+\left(2 \cos \left(\phi-\theta_{13}\right) s_{12} c_{12} \Delta m_{21}^{2}\right)^{2}}\right) \\
& \approx \frac{1}{2}\left(\Delta m_{21}^{2} \cos 2 \theta_{12}+a c_{13}^{2} \pm \sqrt{\left(\Delta m_{21}^{2} \cos 2 \theta_{12}-a c_{13}^{2}\right)^{2}+\left(2 s_{12} c_{12} \Delta m_{21}^{2}\right)^{2}}\right) \\
\sin \psi & =\sqrt{\frac{\lambda_{2}-\lambda_{0}}{\lambda_{2}-\lambda_{1}}}, \quad \text { for }\left|a / \Delta m_{e e}^{2}\right| \ll 1
\end{aligned}
$$

which satisfies

$$
\psi(a) \approx \frac{\pi}{2}-\psi\left(2 \Delta m_{21}^{2} \cos \theta_{12} / c_{13}^{2}-a\right) \quad \text { and } \quad \phi_{N O}(a) \approx \phi_{I O}(a) .
$$

$\psi$ is $\theta_{12}$ in matter and $\left(\lambda_{-}, \lambda_{0}, \lambda_{+}\right) \rightarrow\left(\lambda_{1}, \lambda_{2}, \lambda_{3}\right)$. When $\left|\lambda_{0}-\lambda_{-}\right| \gg 2 s_{12} c_{12} \Delta m_{21}^{2}$ then $\left(\lambda_{1}, \lambda_{2}\right) \approx$ $\left(\lambda_{-}, \lambda_{0}\right)$ or $\left(\lambda_{0}, \lambda_{-}\right)$. If $a=0, \psi=\theta_{12}$ and $\left(\lambda_{1}, \lambda_{2}, \lambda_{3}\right)=\left(-s_{12}^{2} \Delta m_{21}^{2}, c_{21}^{2} \Delta m_{21}^{2}, \Delta m_{e e}^{2}\right)$, thus, in vacuum, $\Delta \lambda_{j k} \equiv \lambda_{j}-\lambda_{k}=\Delta m_{j k}^{2}$. See Appendix for further details.

To calculate the oscillation probabilities, to 0th order, use the above $\Delta \lambda_{j k}$ instead of $\Delta m_{j k}^{2}$ and replace the vacuum MNS matrix as follows ${ }^{3}$

$$
U_{M N S}^{0} \equiv U_{23}\left(\theta_{23}\right) U_{13}\left(\theta_{13}, \delta\right) U_{12}\left(\theta_{12}\right) \Rightarrow U_{M N S}^{M} \equiv U_{23}\left(\theta_{23}\right) U_{13}(\phi, \delta) U_{12}(\psi) .
$$

That is, replace

$$
\Delta m_{j k}^{2} \rightarrow \Delta \lambda_{j k} \quad \theta_{13} \rightarrow \phi, \quad \theta_{12} \rightarrow \psi
$$

it is that simple. $\theta_{23}$ and $\delta$ remain unchanged. Our expansion parameter is $\left|\sin \left(\phi-\theta_{13}\right) s_{12} c_{12} \frac{\Delta m_{21}^{2}}{\Delta m_{e e}^{2}}\right| \leq$ 0.015 , which is small and vanishes in vacuum, so that our perturbation theory reproduces the vacuum oscillation probabilities exactly. A summary of the relevant expressions are given in Fig. 1 and alternative summary using a more conventional notation is given in Appendix II.

\subsection{Higher Orders}

If the 0th order is not accurate enough, going to 1 st order is simple and gives another two orders of magnitude in accuracy. First the eigenvalues $\lambda_{j}$ remain unchanged but the mixing matrix is modified by

$$
U_{M N S}^{M} \Rightarrow V \equiv U_{M N S}^{M}\left(1+W_{1}\right),
$$

where the matrix $W_{1}$ is given by ${ }^{4}$

$$
W_{1}=\sin \left(\phi-\theta_{13}\right) s_{12} c_{12} \Delta m_{21}^{2}\left(\begin{array}{ccc}
0 & 0 & -s_{\psi} e^{-i \delta} / \Delta \lambda_{31} \\
0 & 0 & +c_{\psi} e^{-i \delta} / \Delta \lambda_{32} \\
+s_{\psi} e^{+i \delta} / \Delta \lambda_{31} & -c_{\psi} e^{+i \delta} / \Delta \lambda_{32} & 0
\end{array}\right) .
$$

The $\Delta \lambda_{j k}$ and the $V$-mixing matrix can be used to calculate the oscillation probabilities and improve the accuracy so that $\Delta P<10^{-6}$. The next highest order is also discussed in [1].

\footnotetext{
${ }^{3}$ For the rest of this paper we use the standard parameterization of the MNS matrix for the reader's convenience, as oppose to the parametrization used in [1].

${ }^{4}$ The phase in $W_{1}$ differ from [1] because here we use the standard parameterization of $U_{M N S}$.
} 


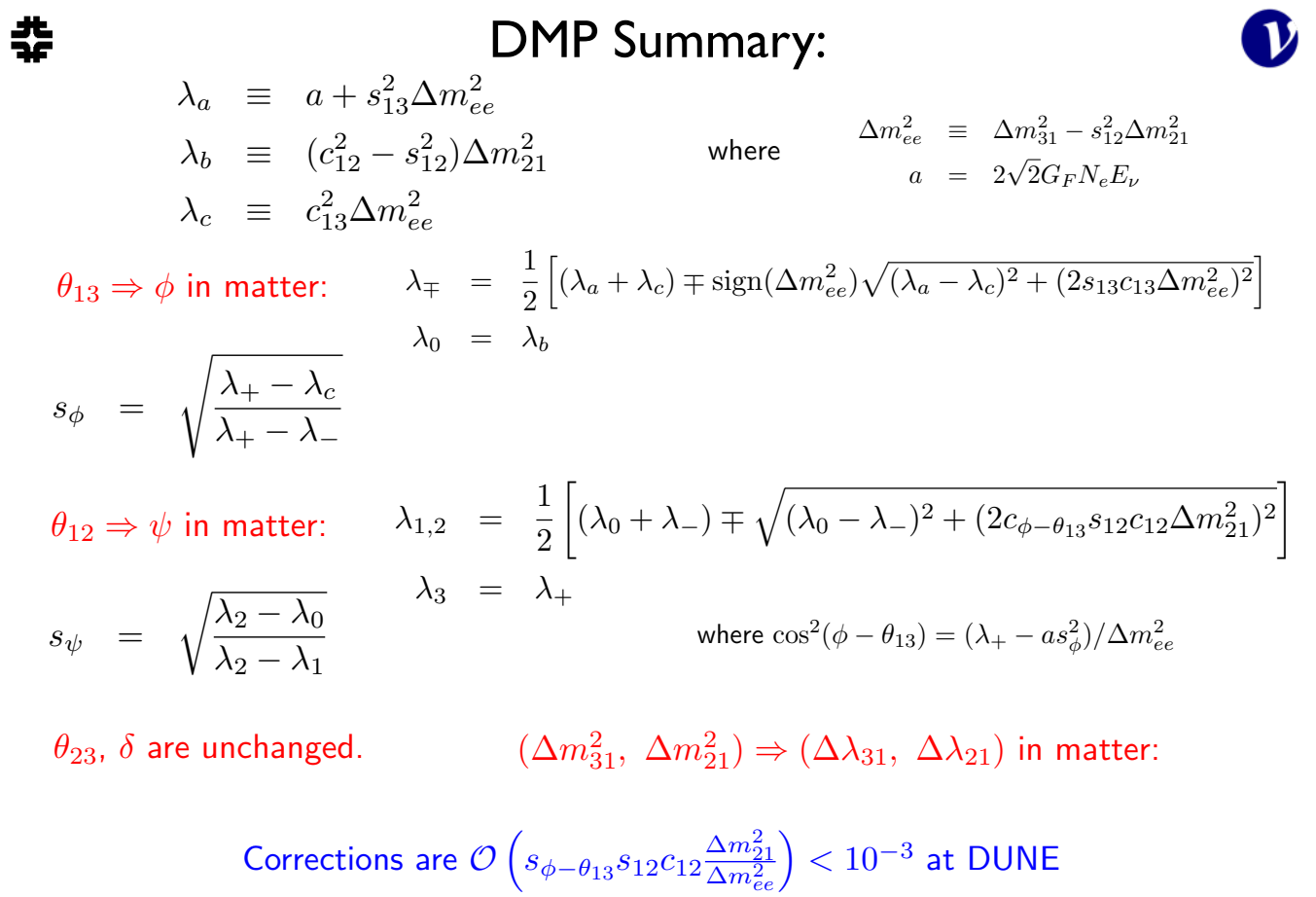

Figure 1: Summary of DMP perturbation theory, 0th order, see [1], for the mixing angles and mass squared eigenvalues in matter. Replacing $\left(\theta_{12}, \theta_{13}, \theta_{23}, \delta, \Delta m_{31}^{2}, \Delta m_{21}^{2}\right)$ with $\left(\psi, \phi, \theta_{23}, \delta, \Delta \lambda_{31}, \Delta \lambda_{21}\right)$ in the vacuum oscillation expressions gives oscillation probability in matter to 0th order. Only 6 square root operations are required to go from the vacuum to matter parameters, not counting the simple $(+,-, *, /)$ operations. No other time consuming operations like sine, cosine, arcsine, arccosine etc are needed.
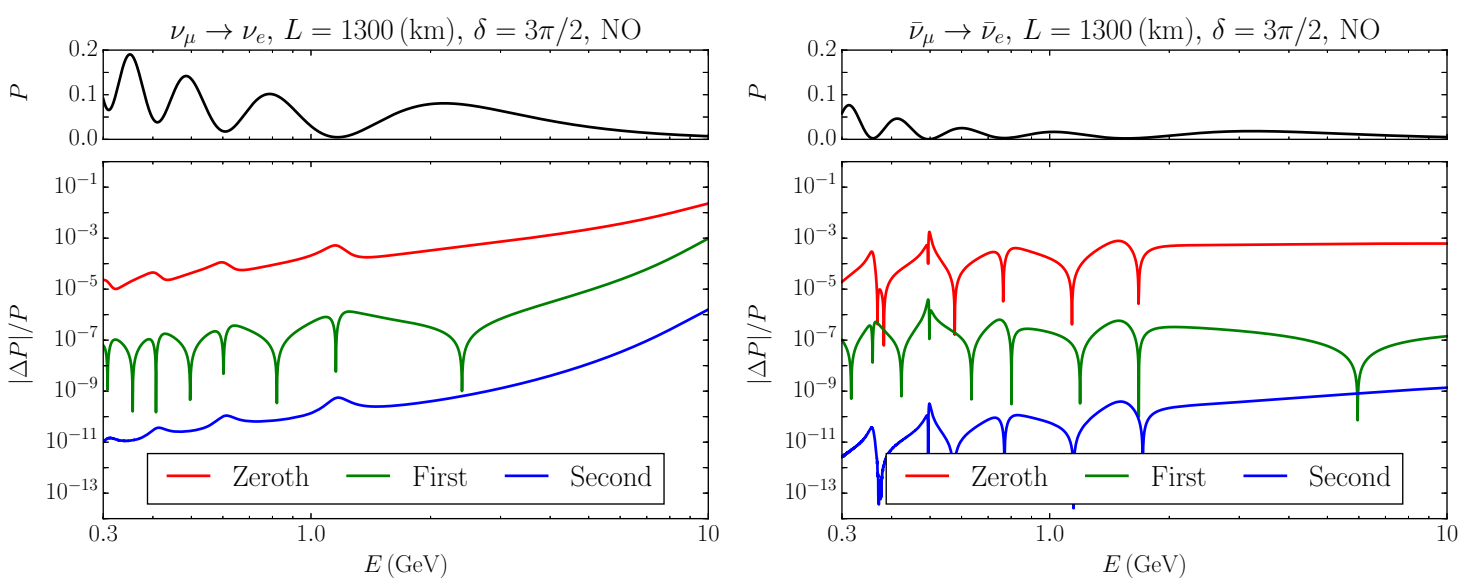

Figure 2: Dune $v_{\mu} \rightarrow v_{e}$ and $\bar{v}_{\mu} \rightarrow \bar{v}_{e}$ appearance probabilities, top panel. The bottom panel, shows the fractional difference between the 0 th, 1 st and 2 nd order approximations to the exact probabilities assuming a constant matter density. 
In Fig. 2 we have compared the exact oscillation probability with our approximation. One sees that the 0th order oscillation probabilities, relevant for the DUNE experiment, have a difference, from the exact calculation, $|\Delta P|<10^{-4}$ and $|\Delta P| / P<10^{-3}$. Higher orders are even more accurate.

\section{Conclusions}

We have summarized our perturbation theory in matter for the neutrino oscillation probabilities, that gives the neutrino mass eigenvalues squared in matter in simple terms only involving the $\sqrt{\cdots}$ function. We also show how the mixing angles in matter can be obtained directly once one knows the matter mass squareds. Higher orders are simple to obtain and increase the accuracy by about two orders of magnitude per order. However, the zeroth order approximations are good enough for all current and future accelerator experiments; T2K, NOvA, DUNE and T2HK/T2HKK due to the uncertainties associated with the matter density profile, height and shape, between neutrino production and detection.

\section{Appendix}

For NO we give approximate expressions for the $\lambda$ 's, in the different regions of interest;

$$
\lambda_{3} \approx \begin{cases}a+s_{13}^{2} \Delta m_{e e}^{2}, & a \gg \Delta m_{e e}^{2} \\ \frac{1}{2}\left(\Delta m_{e e}^{2}+a\right. & \\ \left.\quad+\sqrt{\left(\Delta m_{e e}^{2} \cos 2 \theta_{13}-a\right)^{2}+\left(2 s_{13} c_{13} \Delta m_{e e}^{2}\right)^{2}}\right), & a \approx \Delta m_{e e}^{2} \\ \Delta m_{e e}^{2}+s_{13}^{2} a, & |a| \ll \Delta m_{e e}^{2} \\ c_{13}^{2} \Delta m_{e e}^{2}, & -a \gg \Delta m_{e e}^{2} .\end{cases}
$$

One can obtain similar expressions for $\lambda_{-}$using $\lambda_{-}=\Delta m_{e e}^{2}+a-\lambda_{3}$. Also,

$$
\lambda_{1} \approx \begin{cases}\cos 2 \theta_{12} \Delta m_{21}^{2}, & a \gg \Delta m_{21}^{2} \\ \frac{1}{2}\left(\Delta m_{21}^{2} \cos 2 \theta_{12}+a c_{13}^{2}\right. & \\ \left.-\sqrt{\left(\Delta m_{21}^{2} \cos 2 \theta_{12}-a c_{13}^{2}\right)^{2}+\left(2 s_{12} c_{12} \Delta m_{21}^{2}\right)^{2}}\right), & a \approx \Delta m_{21}^{2} \\ -s_{12}^{2} \Delta m_{21}^{2}+c_{12}^{2} c_{13}^{2} a, & |a| \ll \Delta m_{21}^{2} \\ a+s_{13}^{2} \Delta m_{e e}^{2}, & -a \gg \Delta m_{e e}^{2} .\end{cases}
$$

To obtain $\lambda_{2}$ in all regions of interest, we use $\lambda_{2}=\Delta m_{e e}^{2}+\cos 2 \theta_{12} \Delta m_{21}^{2}+a-\lambda_{3}-\lambda_{1}$;

$$
\lambda_{2} \approx \begin{cases}c_{13}^{2} \Delta m_{e e}^{2}, & a \gg \Delta m_{e e}^{2} \\ \frac{1}{2}\left(\Delta m_{e e}^{2}+a\right. & \\ \left.-\sqrt{\left(\Delta m_{e e}^{2} \cos 2 \theta_{13}-a\right)^{2}+\left(2 s_{13} c_{13} \Delta m_{e e}^{2}\right)^{2}}\right), & a \approx \Delta m_{e e}^{2} \\ \frac{1}{2}\left(\Delta m_{21}^{2} \cos 2 \theta_{12}+a c_{13}^{2}\right. & \\ \left.\quad+\sqrt{\left(\Delta m_{21}^{2} \cos 2 \theta_{12}-a c_{13}^{2}\right)^{2}+\left(2 s_{12} c_{12} \Delta m_{21}^{2}\right)^{2}}\right), & a \approx \Delta m_{21}^{2} \\ c_{12}^{2} \Delta m_{21}^{2}+s_{12}^{2} c_{13}^{2} a, & |a| \ll \Delta m_{21}^{2} \\ \left(c_{12}^{2}-s_{12}^{2}\right) \Delta m_{21}^{2}, & -a \gg \Delta m_{e e}^{2} .\end{cases}
$$

Since $\psi$ and $\phi$ are given in terms of the eigenvalues earlier, approximations for all variables can be easily derived from these $\lambda$ 's. For IO, one can give similar expressions for $\lambda$ 's. 


\section{Appendix II}

We have written everything in terms of the matter eigenvalues, the $\lambda$ 's, since if you know the $\lambda$ 's you can easily calculate the matter mixing angles, $\phi\left(=\widetilde{\theta}_{13}\right)$ and $\psi\left(=\widetilde{\theta}_{12}\right)$, this is summarized in Fig. 1.

However, the mixing angles in matter, which we denote by a $\widetilde{\theta}_{13}$ and $\widetilde{\theta}_{12}$ here, can also be calculated in the following way, using $\Delta m_{e e}^{2} \equiv \cos ^{2} \theta_{12} \Delta m_{31}^{2}+\sin ^{2} \theta_{12} \Delta m_{32}^{2}$, as follows ${ }^{5}$ :

$$
\begin{aligned}
\cos 2 \widetilde{\theta}_{13}= & \frac{\left(\cos 2 \theta_{13}-a / \Delta m_{e e}^{2}\right)}{\sqrt{\left(\cos 2 \theta_{13}-a / \Delta m_{e e}^{2}\right)^{2}+\sin ^{2} 2 \theta_{13}}} \\
& \text { where } a \equiv 2 \sqrt{2} G_{F} N_{e} E \quad \text { is the standard matter potential, and } \\
\cos 2 \widetilde{\theta}_{12}= & \frac{\left(\cos 2 \theta_{12}-a^{\prime} / \Delta m_{21}^{2}\right)}{\sqrt{\left(\cos 2 \theta_{12}-a^{\prime} / \Delta m_{21}^{2}\right)^{2}+\sin ^{2} 2 \theta_{12} \cos ^{2}\left(\widetilde{\theta}_{13}-\theta_{13}\right)}} \\
& \text { where } a^{\prime} \equiv a \cos ^{2} \widetilde{\theta}_{13}+\Delta m_{e e}^{2} \sin ^{2}\left(\widetilde{\theta}_{13}-\theta_{13}\right) \text { is the 13-modified matter potential. }
\end{aligned}
$$

In these two flavor rotations, both $\widetilde{\theta}_{13}$ and $\widetilde{\theta}_{12}$ are in range $[0, \pi / 2]$.

$\theta_{23}$ and $\delta$ are unchanged in matter for this approximation.

The neutrino mass squared differences in matter, i.e. the $\Delta m_{j k}^{2}$ in matter, which we denote by $\widetilde{\Delta m^{2}}{ }_{j k}$, are given by

$$
\begin{aligned}
& {\widetilde{m^{2}}}_{21}=\Delta m_{21}^{2} \sqrt{\left(\cos 2 \theta_{12}-a^{\prime} / \Delta m_{21}^{2}\right)^{2}+\sin ^{2} 2 \theta_{12} \cos ^{2}\left(\widetilde{\theta}_{13}-\theta_{13}\right)} \\
& {\widetilde{m^{2}}}_{31}=\Delta m_{31}^{2}+\frac{1}{2}\left(2 a-3 a^{\prime}+\Delta{\widetilde{m^{2}}}_{21}-\Delta m_{21}^{2}\right) \\
& {\widetilde{m^{2}}}_{32}=\Delta{\widetilde{m^{2}}}_{31}-\Delta{\widetilde{m^{2}}}_{21}=\Delta m_{32}^{2}+\frac{1}{2}\left(2 a-3 a^{\prime}-\Delta{\widetilde{m^{2}}}_{21}+\Delta m_{21}^{2}\right)
\end{aligned}
$$

Note, the same square root appears, as for the $\theta_{12}$ mixing angle in matter ${ }^{6}$. These expressions are valid for both NO, $\Delta m_{31}^{2}>0$ and IO, $\Delta m_{31}^{2}<0$. For anti-neutrinos, just change the sign of $a$ and $\delta$.

If $P_{v_{\alpha} \rightarrow v_{\beta}}\left(\Delta m_{31}^{2}, \Delta m_{21}^{2}, \theta_{13}, \theta_{12}, \theta_{23}, \delta\right)$ is the oscillation probability in vacuum then $P_{v_{\alpha} \rightarrow v_{\beta}}\left(\Delta \widetilde{m^{2}}{ }_{31}, \Delta \widetilde{m^{2}}{ }_{21}, \widetilde{\theta}_{13}, \widetilde{\theta}_{12}, \theta_{23}, \delta\right)$ is the oscillation probability in matter, i.e. use the same function but replace the mass squared differences and mixing angles with the matter values given in eq. 5.1-5.3. The resulting oscillation probabilities are identical to the zeroth order approximation given in Denton, Minakata and Parke, [1].

\footnotetext{
${ }^{5}$ Vacuum values to be used in calculating $\Delta m_{e e}^{2}$.

${ }^{6}$ If $a=0$, then $\widetilde{\theta}_{13}=\theta_{13}$ and since $a^{\prime}=0$ then $\widetilde{\theta}_{12}=\theta_{12}$ and both $\sqrt{\cdots}=1$, also $\widetilde{\Delta m^{2}}{ }_{j k}=\Delta m_{j k}^{2}$ for all $(j, k)$ as required.
} 


\section{Acknowledgements}

This manuscript has been authored by Fermi Research Alliance, LLC under Contract No. DEAC02-07CH11359 with the U.S. Department of Energy, Office of Science, Office of High Energy Physics.

This project has received funding/support from the European Union's Horizon 2020 research and innovation programme under the Marie Sklodowska-Curie grant agreement No 690575. This project has received funding/support from the European Union's Horizon 2020 research and innovation programme under the Marie Sklodowska-Curie grant agreement No 674896.

HM is supported by Instituto Física Teórica, UAM/CSIC in Madrid, via "Theoretical challenges of new high energy, astro and cosmo experimental data" project, Ref: 201650E082.

PBD acknowledges support from the Villum Foundation (Project No. 13164) and the Danish National Research Foundation (DNRF91 and Grant No. 1041811001).

\section{References}

[1] P. B. Denton, H. Minakata and S. J. Parke, "Compact Perturbative Expressions For Neutrino Oscillations in Matter,” JHEP 1606, 051 (2016) doi:10.1007/JHEP06(2016)051 [arXiv:1604.08167 [hep-ph]].

H. Minakata and S. J. Parke, "Simple and Compact Expressions for Neutrino Oscillation Probabilities in Matter,” JHEP 1601, 180 (2016) doi:10.1007/JHEP01(2016)180 [arXiv:1505.01826 [hep-ph]].

[2] H. W. Zaglauer and K. H. Schwarzer, "The Mixing Angles in Matter for Three Generations of Neutrinos and the MSW Mechanism,” Z. Phys. C 40, 273 (1988). doi:10.1007/BF01555889

K. Kimura, A. Takamura and H. Yokomakura, "Exact formulas and simple CP dependence of neutrino oscillation probabilities in matter with constant density,” Phys. Rev. D 66, 073005 (2002) doi:10.1103/PhysRevD.66.073005 [hep-ph/0205295].

[3] J. Arafune and J. Sato, “CP and T violation test in neutrino oscillation,” Phys. Rev. D 55 (1997) 1653 doi:10.1103/PhysRevD.55.1653 [hep-ph/9607437].

J. Arafune, M. Koike and J. Sato, "CP violation and matter effect in long baseline neutrino oscillation experiments,” Phys. Rev. D 56, 3093 (1997) Erratum: [Phys. Rev. D 60, 119905 (1999)] doi:10.1103/PhysRevD.60.119905, 10.1103/PhysRevD.56.3093 [hep-ph/9703351].

M. Freund, "Analytic approximations for three neutrino oscillation parameters and probabilities in matter,” Phys. Rev. D 64, 053003 (2001) doi:10.1103/PhysRevD.64.053003 [hep-ph/0103300].

E. K. Akhmedov, R. Johansson, M. Lindner, T. Ohlsson and T. Schwetz, "Series expansions for three flavor neutrino oscillation probabilities in matter," JHEP 0404 (2004) 078 doi:10.1088/1126-6708/2004/04/078 [hep-ph/0402175]. 